\author{
ANNA ŻURAWSKA \\ ORCID: 0000-0002-5167-6647 \\ Université Nicolas Copernic de Toruń \\ zurawska@umk.pl
}

\title{
HILDEGARDE DE BINGEN, UNE SAINTE POST-SÉCULIÈRE ? LA CLOTTURE DES MERVEILLES DE LORETTE NOBÉCOURT
}

Yves Ledure remarque que la modernité n'est que « la mise en œuvre des Lumières $»^{1}$. Dans cette optique, Ferdinand Buisson rappelle que

[1]a Révolution française fit apparaître pour la première fois dans sa netteté entière l'idée de l'État laïque [...] la délimitation profonde entre le temporel et le spirituel, est entrée dans nos mœurs de manière à ne plus en sortir. [...] rien n'a pu empêcher la société française de devenir, à tout prendre, la plus laïque, la plus séculière de l'Europe ${ }^{2}$.

Cependant, dans les deux dernières décennies, la littérature de ce pays, appelé le plus laïque de l'Europe, manifeste un certain intérêt pour la thématique religieuse (par ex. Gilles Rozier, Moïse fiction [2001], Emmanuel Carrère, Le Royaume [2014], Véronique Olmi, Bakhita [2017]), sans pourtant toujours entretenir un rapport avec l'orthodoxie d'une religion. Lorette Nobécourt appartient à ce groupe d'écrivains qui se réfèrent aux mythes bibliques ou récits hagiographiques et qui réalisent ainsi les postulats de la pensée post-séculière dans la littérature française contemporaine. La spiritualité qui, dans sa réflexion, est étroitement liée à l'acte de création artistique, notamment à l'écriture ${ }^{3}$, constitue le sujet principal de ses œuvres récentes (à partir de Substance, 2001), ce dont témoigne aussi son treizième récit, La Vie

${ }^{1}$ Y. Ledure, Sécularisation et spiritualité. Approche anthropologique du christianisme, Lessius, Bruxelles 2014, pp. 32-33.

${ }^{2}$ F. Buisson, « La laïcité selon Ferdinand Buisson », L'Esprit républicain, 2 mai 2007, <http:// action-republicaine.over-blog.com/article-6732463.html> [consulté le 3.01.2017].

${ }^{3}$ Cf. C. Zoulim, «Vivre sa foi au XXI ${ }^{\mathrm{e}}$ siècle : Lorette Nobécourt, ou les mystères du verbe », [dans :] J. Bielska-Krawczyk, A. Żurawska (dir.), Homo spiritualis of the $20^{\text {th }}$ and the $21^{\text {st }}$ Centuries / Homo spiritualis aux XXe et XXIe siècles, Wydawnictwo Libron, Kraków 2016, pp. 139-150. 
spirituelle, publié en $2017^{4}$. Par contre, ses premiers textes ont souvent un caractère autofictif et explorent la thématique liée au corps humain en exprimant ainsi la souffrance de l'artiste. Or, depuis son enfance, Nobécourt a dû faire face à des maladies cutanées qui ont été pour elle à l'origine d'une douleur physique, mais aussi de nombreuses humiliations, d'un sentiment d'altérité et d'un rejet qu'elle a subi de la part de sa famille et de ses collègues. C'est dans la littérature qu'elle a trouvé le salut, l'espoir et la force vitale. C'est aussi la littérature qui a généré sa réflexion sur la dimension spirituelle de l'humain (non nécessairement liée à une religion institutionnalisée) et sur sa richesse intérieure. Le roman La Clôture des merveilles. Une vie d'Hildegarde de Bingen (2013), objet de la présente analyse, est représentatif de la tendance métaphysique de l'œuvre de Nobécourt.

L'objectif de cet article est de proposer une réflexion sur les motivations qui ont amené l'écrivaine française contemporaine à choisir sainte Hildegarde, Docteur de l'Église, pour protagoniste de son roman. Par conséquent, nous nous interrogerons sur la façon dont ce personnage a été présenté dans le texte, ainsi que sur la qualité de la relation entre la sainte telle qu'elle apparaît dans le récit, et la narratrice. Cela nous amènera également à examiner le genre de spiritualité qu'elles représentent. Enfin, nous nous poserons la question de savoir comment le lecteur laïque contemporain devrait comprendre le lexème clôture, qui apparaît déjà dans le titre du roman.

Les outils théoriques élaborés par Charles Taylor, surtout sa conception du " lieu de plénitude » (ang. place of fullness), et par John A. McClure serviront à analyser le texte et à répondre aux questions posées ci-dessus.

\section{NOSTALGIE}

Dans La Clôture des merveilles, Nobécourt recrée l'histoire d'Hildegarde de Bingen $^{5}$ qui, dans le texte, est appelée H. Cette simple initiale est probablement plus pratique que le nom entier, mais il se peut aussi que de cette manière la narratrice veuille marquer une nette distinction entre le personnage historique de sainte Hildegarde et $\mathrm{H}$., hérö̈ne fictive qu'elle a créée ${ }^{6}$. De plus, la réduction du prénom

${ }^{4}$ En 2018, elle a publié chez Le Cerf encore deux textes : Le Poème perdu et Vivant Jardin.

${ }^{5} \mathrm{Au}$ sujet de la biographie et de la spiritualité d'Hildegarde, voir : E. Wiater, Hildegarda z Bingen. Mistyczka z charakterem, Wydawnictwo eSPe, Kraków 2016 ; H. Multhaupt, W Jego świetle. Opowieść o Świętej Hildegardzie z Bingen, trad. M. Dobija, Wydawnictwo Esprit, Kraków 2015 ; Modlitwy Hildegardy z Bingen, trad. K. Wiwer, Wydawnictwo Astraia, Kraków 2008 ; Hildegarda z Bingen, Wizje, czyli poznaj drogi Pana, trad. J. Łukaszewska-Haberkowa, Wydawnictwo Benedyktynów Tyniec, Kraków 2011.

${ }^{6}$ Cette thèse est aussi confirmée par le choix grammatical de l'article indéfini dans le sous-titre du roman (Une vie d'Hildegarde de Bingen), qui suggère l'invention de l'écrivaine concernant la vie de sainte Hildegarde et sa liberté dans le traitement des faits biographiques. 
à l'initiale rend ce personnage plus mystérieux, comme si son identité était encore à découvrir au fil de la narration.

Le récit n'est pas une simple biographie de la sainte, bien que des éléments biographiques soient présents dans le texte et que la narration respecte l'ordre linéaire et chronologique - si l'on ne compte pas de longues séquences digressives qui sont de nature spirituelle, voire mystique et qui dépassent le cadre temporel du récit en se situant en dehors de l'histoire racontée, créant ainsi un nouvel espace-temps, lieu de rencontre de deux femmes, H. et la narratrice. Plus que de la biographie, le roman s'approche de l'histoire d'une âme, ou d'un récit raconté de l'intérieur, dans la perspective de la réalité spirituelle. Vu l'attitude personnelle de la narratrice face à la protagoniste, il semble justifié de se poser la question des facteurs qui ont amené l'écrivaine contemporaine à choisir un personnage de la pléiade des saints médiévaux. Quelle est la raison de ce retour post-séculier à la tradition chrétienne ?

La narratrice avoue explicitement à la première page du roman qu'elle ne s'identifie ni à la religion, ni à l'idéologie de la laïcité, mais en même temps, elle se sent attachée à Hildegarde. Elle dit : « je suis de sa clôture » ${ }^{7}$.

Cette attitude entre les deux - la religion et la laïcité — ou mieux, cette situation existentielle qui détermine la place de la narratrice " à l'intersection des systèmes de croyance sacrés et séculiers ${ }^{8}$, est caractéristique des écrivains post-séculiers et des personnages qu'ils créent ${ }^{9}$. Dieu a été d'ailleurs partiellement évacué du couvent d'H., et s'Il apparaît dans le roman (par exemple comme une personne de la Sainte Trinité), Il ne correspond pas nécessairement à une vision conforme à la doctrine, mais est présenté en tant que personnage, certes, inspiré par le christianisme, mais possédant des propriétés particulières. L'écart qui se crée entre les deux attitudes face à la foi, représentées respectivement par Hildegarde et la narratrice, est donc manifeste : pour l'une, elle est essentielle et obligatoirement liée à l'institution de l'Église, alors que le rapport à la foi de l'autre est très individualisé et peu orthodoxe ${ }^{10}$. En effet, cet écart n'est qu'une conséquence de la confrontation du Moyen Âge et de la modernité.

${ }^{7}$ L. Nobécourt, La Clôture des merveilles. Une vie d'Hildegarde de Bingen, Éditions Grasset \& Fasquelle, Paris 2013, p. 11.

8 J.A. McClure, Pótwiary. Literatura postsekularna w czasach Pynchona i Morrison, trad. T. Umerle, Wydawnictwo Uniwersytetu Jagiellońskiego, Kraków 2016, p. 29. Si ce n'est pas indiqué autrement, les traductions de textes du polonais en français sont de l'auteure de l'article.

${ }^{9}$ Ibidem, pp. 32-33 : « La religiosité post-séculière ne veut avoir rien en commun avec les programmes et scénarios universels caractéristiques des systèmes du pouvoir sacrés. [...] en acceptant sa position incertaine, elle est prête - pour des raisons bien plus profondes, dépassant le pur pragmatisme - à écouter les autres et à communiquer avec eux dans la sphère publique et pluraliste. Elle renonce en même temps aux solutions universelles et aux stratégies triomphalistes du sécularisme dogmatique avec leur histoire sanglante ».

${ }^{10} \mathrm{Au}$ sujet de la religion personnelle et du rôle de l'individualisme dans le processus de la sécularisation, voir Ch. Taylor, La diversité de l'expérience religieuse aujourd'hui. William James revisité, trad. J.-A. Billard, Éditions Bellarmin, Montréal 2003, pp. 18-19, 76-104. 
L'incipit du roman apporte une première esquisse de réponse à la question posée ci-dessus : la sainte est pour la narratrice un personnage fascinant qui éveille aussi bien l'admiration que la nostalgie et qui représente - à moins que la thèse ne soit trop osée - ce que, suivant la pensée de William James, Taylor appelle « le génie religieux ${ }^{11}$. L'interrogation des premières pages du texte en témoigne : « Où sont les Hildegarde du XXI ${ }^{\mathrm{e}}$ siècle $[\ldots]$ ? $»^{12}$. Cette question relie, de manière très simple, deux perspectives temporelles (médiévale et contemporaine), ce qui est caractéristique des narrations néo-médiévales et donc aussi néo-monastiques. Qui plus est, ce récit qui réalise l'idée du néo-monachisme, dans l'acception que lui accorde McClure, bien qu'il ne réponde pas à tous les critères demandés pour ce type de narration $^{13}$, souligne l'importance du retour à la tradition bénédictine et valorise par cela les monastères en tant qu'endroits protégeant les valeurs, abritant ceux qui en ont besoin, favorisant la métanoïa ${ }^{14}$, enfin, en tant que centres d'ascèse et de méditation ${ }^{15}$.

La question « Où sont les Hildegarde du XXI ${ }^{\mathrm{e}}$ siècle $[\ldots]$ ? » ${ }^{16}$ semble alors exprimer une grande nostalgie. Quel est pourtant son objet?

\section{PLÉNITUDE}

Dans son ouvrage L'Âge séculier, Taylor explique les raisons du processus de sécularisation dans le monde contemporain. Il expose trois significations de la sécularité : la première concerne les institutions étatiques, donc l'espace public, la deuxième doit être comprise comme abandon des pratiques religieuses, la

11 Ibidem, p. 11 : «"Nous chercherons plutôt les expériences originales qui ont servi de modèle à tous ces sentiments suggérés et à ces pratiques stéréotypées. Or, l'on ne peut rencontrer de telles expériences que chez des individus pour qui la religion existe, non point émoussée par l'habitude, mais plutôt à l'état aigu de fièvre. Mais de tels individus sont des 'génies' dans l'ordre religieux". Nous voyons ici l'idée que se fait James de la vie religieuse, de son origine et de sa perpétuation : certains individus ont une expérience religieuse originale et puissante qui se trouve transmise alors par une institution donnée...».

${ }^{12}$ L. Nobécourt, op. cit., p. 16.

${ }^{13}$ Le récit n'accomplit pas la condition qui prévoit la fondation par des personnages littéraires de communautés post-séculières qui seraient nouvelles, locales, spirituelles. Celles-ci s'abriteraient dans d'anciens couvents et, s'écartant du monde, s'opposeraient à l'ordre dominant (ou à des ordres au pluriel : d'une part séculier, de l'autre doctrinal). Quoi qu'il en soit, l'importance du couvent ou de la clôture (évoquée déjà au niveau du titre du roman) ainsi que le fait qu'Hildegarde elle-même a été fondatrice de nombreuses communautés de moniales au Moyen Âge fait que le roman répond, dans une certaine mesure, à l'idée de McClure. Le retrait du monde et l'opposition aux ordres évoqués ci-dessus sont aussi caractéristiques de Nobécourt.

${ }^{14}$ Cf. J.A. McClure, op. cit., pp. 37-41. McClure rappelle à cette occasion l'importance de la spiritualité franciscaine dans la littérature réalisant l'idée du néo-monachisme.

${ }^{15}$ Sur l'importance des monastères au Moyen Âge et le rôle qu'ils devraient jouer aujourd'hui, voir : B. Griffiths, Złota nić. Chrześcijański aśram, trad. J. Mroczkowska et P. Mroczkowski, Znak, Kraków 1974, pp. 14-15.

${ }^{16}$ L. Nobécourt, op. cit., p. 16. 
troisième est la plus complexe et constitue l'objet d'une étude plus approfondie de sa part. La sécularité dans sa troisième acception consiste dans un « changement [...] qui nous mène d'une société dans laquelle il était virtuellement impossible de ne pas croire en Dieu, à une société où la foi, y compris pour le croyant le plus inébranlable, est une possibilité parmi d'autres $»^{17}$.

Comme le rappelle Taylor, dans l'Europe médiévale, « il était virtuellement impossible de ne pas croire en Dieu ${ }^{18}$. De plus, dans le cas d'Hildegarde, le credo chrétien se réalisait derrière les murs d'un monastère, c'est-à-dire que sa vie était entièrement soumise à l'unique option possible, elle était marquée par sa décision radicale d'obéir à Dieu. Dans le monde occidental d'aujourd'hui où vit Nobécourt, la foi, d'après l'explication de Taylor, n'est qu'une des nombreuses options, et ce n'est pas nécessairement l'option la plus évidente. Dans sa création littéraire d'Hildegarde, la narratrice adopte la perspective contemporaine, donc sa perception de la sainte est sélective : elle admire ses qualités en les séparant cependant de sa foi, essence de la vie d'Hildegarde, ou en modifiant les vérités chrétiennes et en les adaptant à sa propre interprétation de la mystique médiévale. En contemplant le personnage d'Hildegarde, la narratrice remarque surtout son amour, sa liberté et sa beauté. Ces valeurs, d'ailleurs les plus souvent évoquées dans le texte, ne sont pourtant pas toujours considérées comme vertus dues à une profonde relation avec Dieu, mais comme preuve de l'accomplissement qui peut se réaliser aussi bien dans un espace laïque. Dans ce cas-là, la vie est envisagée comme vécue consciemment et sensément. La narratrice constate : «En m'emparant d'une telle figure, [...] [je veux] dire haut et clair que la vie vivante est la seule. Et poser la beauté et la liberté comme les deux conditions sine qua non de son accomplissement. Voilà ce que je suis » ${ }^{19}$.

Taylor remarque :

[n] ous considérons tous que nos vies, et / ou les lieux où nous vivons nos vies, ont une certaine forme morale et spirituelle. Quelque part, dans certaines activités ou conditions, se niche une plénitude, une richesse : en ce lieu (activité ou condition), la vie nous apparaît plus pleine, plus riche, plus profonde, plus digne d'intérêt, plus admirable, plus fidèle à ce qu'elle doit être. C'est peut-être un lieu où se déploie une puissance : nous faisons souvent ici l'expérience de quelque chose de profondément émouvant, susceptible de nous inspirer. Peut-être cette sensation de plénitude ne peut-elle être entraperçue qu'au loin ; nous ressentons une puissante intuition de ce que serait la plénitude — paix, complétude... - si nous pouvions y accéder ou si nous étions capables d'agir à ce niveau, c'est-àdire avec intégrité, générosité, abandon ou oubli de soi $^{20}$.

Dans la suite de sa réflexion, le philosophe distingue trois attitudes face au lieu de plénitude : la première, c'est la possibilité de vivre réellement l'expérience de la plénitude (soit dans la relation avec l'Être transcendant, soit dans le rapport

\footnotetext{
17 Ch. Taylor, L’Âge séculier, trad. P. Savidan, Seuil, Paris 2011, pp. 15-16.

18 Ibidem.

${ }^{19}$ L. Nobécourt, op. cit., p. 15. L'italique est dans l'original.

${ }^{20}$ Ch. Taylor, L'Âge séculier, pp. 18-19.
} 
avec la Nature, soit dans la situation où l'homme ressent en son for intérieur une certaine force vitale qui pénètre toutes choses, mais dont la provenance reste pour lui obscure ou énigmatique), la deuxième est une sensation d'impuissance, d'incapacité, d'exil, ou encore, la situation où " nous perdons le sens du lieu où se trouve cette plénitude, voire de ce en quoi consiste cette plénitude $»^{21}$, finalement, la troisième est une condition intermédiaire, c'est-à-dire « une vie stable, voire routinière, qui nous voit accomplir des tâches qui ont pour nous un sens - tâches qui, par exemple, contribuent à notre bonheur ordinaire $»^{22}$.

Il semble que l'objet de la nostalgie exprimée par la narratrice du roman de Nobécourt soit, en effet, « la plénitude ». Son attitude face à elle correspondrait à la première signification décrite par Taylor comme la réelle expérience de la plénitude (« quand la réalité ordinaire est "abolie" et que quelque chose de terriblement autre apparaît » ${ }^{23}$ ). La question « Où sont les Hildegarde du XXI" siècle $[\ldots]$ ? $»^{24}$ exprimerait donc la nostalgie de la plénitude dans le contexte d'un appauvrissement spirituel de notre époque, du vide que la narratrice remarque dans le monde ambiant et auquel elle ne veut pas consentir. C'est la nostalgie de « la vie vivante », vécue dans sa pleine dimension ou ses dimensions : spirituelle, intellectuelle, artistique, etc. L'Occident sécularisé s'avère du coup insuffisant, ce qui constitue d'ailleurs un trait majeur du post-sécularisme ${ }^{25}$. Malgré un tel diagnostic de notre époque, comprise comme réalité privée de valeurs spirituelles et dénudée de mystère, la narratrice semble avoir accès à la plénitude et en même temps partager cette expérience avec Hildegarde. L'affinité des expériences fait que les obstacles (temporels, religieux) mentionnés ci-dessus disparaissent parce qu'elle se réalise dans un espace spirituel et mystique, mais non nécessairement religieux. L'expérience partagée rend possible une forte identification de la narratrice avec le personnage décrit : «Et quel bonheur d'écrire sur ce qu'on aime! Non pas Hildegarde de Bingen, mais ce dont elle témoigne, et dont ma vie n'a d'autre ambition que de témoigner aussi. Enfant, c'est cela que j'aimais chez les saints, sans savoir le nommer : l'insoumission $»^{26}$. Ainsi est-il très probable que $\mathrm{H}$. n'est qu'un alter ego de la narratrice, qui est un alter ego de Nobécourt elle-même.

21 Ibidem, p. 21.

22 Ibidem, p. 22.

${ }^{23}$ Ibidem, p. 20.

${ }^{24}$ L. Nobécourt, op. cit., p. 16.

${ }^{25}$ Cf. Ch. Taylor, L’Âge séculier; T. Sławek, « Ratujące niebezpieczeństwo postsekularyzmu. Słowo wstępne ", [dans :] Drzewo poznania. Postsekularyzm w przekładach i komentarzach, P. Bogalecki, A. Mitek-Dziemba (dir.), Wydawnictwo FA-art, Uniwersytet Śląski, Katowice 2012, pp. 9-24 ; J.A. McClure, op. cit., pp. 20-24, et suiv.

${ }^{26}$ L. Nobécourt, op. cit., pp. 11-12. 


\section{VIVRE LA PLÉNITUDE}

Il faudrait cependant rester prudent en formulant la conclusion que les différences dans la manière de vivre la plénitude des deux femmes ont été supprimées. Comme l'observe Taylor :

Le contraste majeur et évident se manifeste dans le fait que, pour les croyants, la représentation de ce lieu de plénitude requiert une référence à Dieu, c'est-à-dire à quelque chose qui se situe au-delà de la vie humaine et / ou de la nature, tandis qu'il n'en va pas de même pour les non-croyants. Ils laisseront ouverte cette représentation ou comprendront la plénitude en la rapportant à une potentialité des êtres humains comprise d'un point de vue naturel ${ }^{27}$.

Le roman considère la plénitude vécue par sainte Hildegarde en tant que preuve d'une potentialité de l'être humain qui s'articule - aussi pour la narratrice/l'écrivaine elle-même - à deux niveaux : à travers l'écriture, donc l'acte de création, et grâce à ce qui est appelé « viridité ${ }^{28}$ (donc une vie vivante, une vie vraie, une force vitale). Pour Nobécourt, comme l'indiquent sa biographie et son œuvre, l'écriture est essentielle : elle devient, d'un côté, une sorte de thérapie, de remède apaisant sa douleur aussi bien physique que morale, résultat des humiliations subies, des conséquences psychologiques de sa maladie. Elle le confirme dans une phrase poignante : «On n'écrit jamais par loisir mais pour ne pas mourir $»^{29}$. Ce propos ne devrait pas être interprété en tant que non omnis moriar, mais bien littéralement : l'écriture est une activité vitale pour l'artiste, elle lui permet de continuer à vivre. La souffrance est donc une expérience qui reste en un rapport direct et très étroit avec le corps qui devient Verbe ${ }^{30}$. L'acte de création a le pouvoir de transformer ce qui est charnel, matériel, physique, en une substance spirituelle.

D'un autre côté, c'est, en effet, la littérature qui donne accès à la plénitude. La même attitude est observée par la narratrice chez Hildegarde : " La singularité de $\mathrm{H}$. se tient dans cette double qualité : la chair tourmentée et l'espérance du verbe $»^{31}$. Nombreuses sont les références à l'acte d'écriture comparé au ciel ouvert, à la fécondité, à une terre inconnue qui se trouve à l'intérieur de l'homme, à la profondeur du silence, etc. ${ }^{32}$ Cependant, en analysant ce que l'écriture représente

${ }^{27}$ Ch. Taylor, L'Âge séculier, p. 24.

${ }^{28}$ Le lexème « viridité » ne semble pas être employé par Nobécourt seulement dans son sens premier (qualité de ce qui est vert), mais elle élargit sa signification comme s'il s'agissait d'un néologisme, d'une sorte de mot-valise composé de « vie », « virilité », « verdure » et suggérant une vitalité, vigueur ou énergie particulière.

${ }^{29}$ L. Nobécourt, op. cit., p. 67.

${ }^{30}$ Małgorzata Grzegorzewska propose, elle aussi, de réfléchir sur la littérature dans le contexte du Verbe (Logos) incarné et d'examiner les relations qui se créent entre la pensée, le verbe et le corps (cf. M. Grzegorzewska, Światłocienie. Osiem odsłon Stowa w literaturze brytyjskiej od Hopkinsa do Hughesa, Homini, Tyniec Wydawnictwo Benedyktynów, Kraków 2016, p. 12).

${ }^{31}$ L. Nobécourt, op. cit., p. 48.

32 Ibidem, pp. 51, 63, 131, et suiv. 
pour Hildegarde, la narratrice - et dans ce contexte, on peut risquer qu'il s'agit de Nobécourt elle-même - partage, en réalité, sa propre expérience d'écriture avec le lecteur. Celle-ci a des qualités d'une expérience religieuse : «H. aperçoit qu'écrire délivre. Écrire perce. Assainit. Nettoie. Fore. Met à jour. Écrase la peur. Transporte hors de soi. Et guérit $»^{33}$. Parfois, l'écriture ressemble à une prière contemplative ou à une expérience mystique où l'homme s'oublie lui-même, c'est-à-dire meurt à soi et au monde ( $"$ Et ainsi l'on meurt à soi pour assumer de naître au verbe ${ }^{34}$ ). Une autre fois, l'écriture permet de voir plus (« [q]ue voir c'est écrire, et qu'écrire c'est voir $»^{35}$ ). Hildegarde est aussi comparée à un outil dans les mains du Verbe, les œuvres de la sainte sont Ses œuvres, et son verbe est le Verbe. Le choix du lexème Verbe (écrit avec une majuscule) au lieu du lexème Dieu semble intentionnel. Le Verbe peut, évidemment, renvoyer à Dieu (surtout à l'incipit de l'Évangile selon Saint Jean), ce qui est tout à fait naturel et fondé dans le contexte de la réflexion sur la vie d'une sainte ; mais le Verbe en lui-même est ici vénéré comme une divinité (qui n'a pourtant pas besoin d'Églises, comme le souligne la narratrice ${ }^{36}$ ). Présentant la première étape de l'œuvre de Nobécourt, Constance Zoulim remarque que, déjà dans cette période-là, l'écrivaine « emprunte [...] le chemin du verbe, choisit de le servir, comme on entre en religion. [...] Le verbe apparait comme le lieu de communion avec le divin et donc de l'expérience mystique $»^{37}$. Le lecteur assiste donc à une sacralisation ${ }^{38}$ de l'acte d'écriture ${ }^{39}$, ou celui-ci est devenu espace de fusion de sacrum et de profanum, ce qui permet d'adopter une approche universalisante envers les deux femmes : le Verbe est essentiel pour les deux, bien que pour l'une, il incarne toujours l'idée de Dieu et que pour l'autre, il doive être identifié au mystère, à la profondeur de la vie, à la viridité évoquée tout à l'heure. Qui plus est, pour l'une, l'écriture n'est qu'un outil sur le chemin qui mène à la pleine et profonde communion avec Dieu, tandis que pour l'autre, elle est le moyen de réconciliation avec elle-même, la clé de l'intérieur. À part la sacralisation du Verbe, la narration
33 Ibidem, p. 68.
${ }^{34}$ Ibidem, p. 67.
35 Ibidem, p. 61.
${ }^{36}$ Ibidem, p. 74.
${ }^{37}$ C. Zoulim, op. cit., p. 144.

${ }^{38}$ Elle propose, sur son site Internet, des ateliers littéraires intitulés « En vivant, en écrivant » qui ressemblent à des retraites quasi religieuses. Le champ lexical du sacré, du spirituel (par ex. " C'est une aventure sacrée », "Clôture dont les portes fermées ouvrent à l'intérieur de soi », « le chemin spirituel ») domine dans la description de ces ateliers qui se divisent en trois sessions qu'il faut suivre dans l'ordre (comme une sorte d'initiation). On y annonce explicitement que le Divin est dans l'écriture : «l'enjeu n'est pas d'apprendre à écrire mais de s'approcher de sa propre langue, unique dans tout l'univers, singulière, par laquelle se guérir, un beau jour être heureux. En ce sens, l'écriture est thérapeutique » [l'italique se trouve dans l'original]. Les retraites se déroulent dans des endroits silencieux et isolés, elles proposent une approche holistique, prenant en compte non seulement le côté spirituel de l'homme, mais aussi le régime alimentaire $(<\mathrm{http}: / /$ laurencenobecourt. com/?page_id=2977> [consulté le 3.11.2018]).

39 Dans ce contexte, il serait peut-être intéressant d'examiner aussi la relation, s'il y en a une, entre Nobécourt et les symbolistes français. 
met également en relief la dimension spirituelle de l'art compris dans un sens très large (Hildegarde de Bingen excellait dans différents domaines de l'art). Selon la narratrice, l'art « est la manifestation physique du ciel qui est en nous $»^{40}$.

L'écriture en elle-même est déjà ce lieu de plénitude et en même temps, elle donne la possibilité de l'exprimer. Reste pourtant à analyser la question du langage qui répondrait aux exigences de l'articulation de cette expérience.

\section{LE LANGAGE FACE À L’EXPÉRIENCE MYSTIQUE}

Dans le contexte de la valorisation de l'activité d'écriture, il faudrait accorder une attention particulière au langage et à la structure du texte. Celui-ci abonde en références à la Bible et en citations puisées dans l'œuvre d'Hildegarde qui alternent avec la part fictive du roman. À part les renvois explicites aux textes sacrés, le langage lui-même semble imiter par moments la stylistique biblique ${ }^{41}$, sans renoncer pourtant à la recherche de nouvelles formes d'expression.

L'approche créative face à la langue préexistante peut servir à forger un outil d'articulation de l'expérience de la plénitude (en témoignent les néologismes imaginés par Nobécourt et la lingua ignota créée par Hildegarde ainsi que le langage de ses visions dans Scivias). Taylor insiste sur le fait que l'articulation de l'expérience de la plénitude peut s'avérer nécessaire :

Nous nous trouvons alors profondément émus, et en même temps intrigués et bouleversés. Nous luttons pour exprimer ce que nous avons vécu. Si nous réussissons à le formuler, ne serait-ce que partiellement, nous en sommes soulagés, comme si le pouvoir de l'expérience se trouvait accru du fait qu'il aura été précisé, exprimé et par là même libéré. Cela peut nous aider à donner une direction à nos vies ${ }^{42}$.

Le roman La Clôture des merveilles est probablement un essai d'articulation de l'expérience de la plénitude.

Faisant de même appel à la notion de « libération », Tadeusz Sławek voit la nécessité de la recherche d'un nouveau langage ou d'un nouveau style d'expression. La réflexion qu'il appelle latérale au sécularisme et que le roman de Nobécourt représente sûrement, vise, selon lui,

à libérer l'expérience religieuse de la soumission à l'idéologie d'une doctrine, et cela ne devrait donc pas étonner qu'elle cherche, sans être sûre du résultat, un autre style d'expression. [...] Il s'agit donc de rendre aux mots et aux formes d'expression la force qu'ils avaient perdue en restant au service d'une doctrine et y cherchant abri et sécuritét ${ }^{43}$.

${ }^{40}$ L. Nobécourt, op. cit., p. 144.

${ }^{41}$ McClure remarque : «Les discours sacrés resurgissent sans cesse dans les romans post-séculiers. Ils déterminent les expériences des protagonistes et leurs interprétations, ils apparaissent dans les pensées et propos des personnages et fournissent des catégories pour décrire et juger les univers habités par eux » (J.A. McClure, op. cit., p. 17).

${ }^{42}$ Ch. Taylor, L'Âge séculier, p. 21.

${ }^{43}$ T. Sławek, op. cit., p. 23 : « do uwolnienia doświadczenia religijnego od podporządkowania zideologizowanej doktrynie, nie dziwi także, że będzie z lepszym lub gorszym skutkiem poszukiwała 
Aussi bien sainte Hildegarde dans son monde enchanté que Nobécourt dans la réalité contemporaine cherchent un langage nouveau, capable de nommer leurs expériences ${ }^{44}$.

La réflexion sur la recherche des formes d'expression adéquates reste en rapport direct avec l'acte de création lui-même. Les métaphores illustrant l'activité d'écriture (très nombreuses, comme cela a été déjà démontré) se réfèrent plus souvent à Dieu que les autres parties du texte. Mais la vision de Dieu dans le roman est sélective : Son activité se limite surtout à énoncer le Verbe qui résonne dans le silence de la clôture du cœur humain. C'est donc Dieu qui se sert du langage, et celui-ci accomplit parfaitement sa fonction performative (comme dans le Livre de la Genèse ou dans le premier chapitre de l'Évangile selon Saint Jean). C'est aussi Dieu qui fait naître le verbe dans l'intérieur profond de l'homme.

Le langage est aussi compris comme métaphore de la vie ou garantie d'une vraie vie, ou encore comme chemin menant « à la béatitude promettant davantage de vie $»^{45}$. La narratrice a recours à la lingua ignota en l'identifiant à « un chemin de vie $»^{46}$. Ce ne sont donc pas les propriétés grammaticales ou syntaxiques du langage d'Hildegarde qui l'intéressent le plus, mais le fait qu'elle emploie un code secret appartenant à une autre réalité qui reste cachée. Le fait de posséder une telle langue (selon la narratrice, chaque humain la porte en soi) oblige l'homme à répondre à la vocation pour écrire, et le rôle de l'écrivain ou du poète est de déchiffrer le « langage de Dieu ${ }^{47}$.

\section{LA CLÔTURE, LIEU DE PLÉNITUDE}

L'expérience de la plénitude a lieu dans un endroit concret, dans l'isolement, dans la solitude, loin du monde et de son fracas : « Nulle fenêtre, nulle porte à cette enceinte où s'ébauche une plénitude qu'elle [Hildegarde] devine, la source d'un secret qui la bouleverse : cette certitude d'aimer et d'être aimée en retour ${ }^{48}$.

Le mot clôture du titre du roman peut être compris aussi bien de manière littérale que métaphorique. D'une part, l'intitulé renvoie à l'univers médiéval, enchanté et monastique, à l'époque des cathédrales et des croisades. De l'autre, la clôture est synonyme du monachisme intérieur où résident la beauté et les

\footnotetext{
innego stylu wypowiedzi. [...] Chodzi więc o przywrócenie słowu i formom wypowiedzi siły, którą utraciły, szukając schronienia w służbie bezpiecznej doktrynie ».

${ }^{44} \mathrm{Au}$ sujet de la tension entre la nature sociale du langage et l'articulation de l'expérience individuelle, voir : Ch. Taylor, La diversité de l'expérience religieuse aujourd'hui..., pp. 30-33.

45 A. Bielik-Robson, ,Na pustyni”. Kryptologie późnej nowoczesności, cité après : T. Sławek, op. cit., p. 16 : « ku błogosławieństwu obiecującemu więcej życia ».

${ }^{46}$ L. Nobécourt, op. cit., p. 141.

47 Ibidem, p. 138.

48 Ibidem, p. 41.
} 
merveilles. En effet, l'homme est ce monastère habité par Dieu ${ }^{49}$ dans le cas de sainte Hildegarde, ou, à prendre en compte la perspective post-séculière représentée par Nobécourt, il est un temple de vie ${ }^{50}$. Quel que soit le point de vue adopté - religieux ou post-séculier —, l'homme est invité à se retirer du monde, à habiter avec soi-même (habitare secum), tout comme Hildegarde qui « a appris à habiter entièrement qui elle est $\rangle^{51}$; il est invité à apprécier la solitude et le silence (comme les moines qui savent garder le silence ${ }^{52}$ et rester à l'écoute). Le modèle de vie monastique est présenté dans le roman comme moyen assurant l'expérience de la profondeur, de la plénitude ${ }^{53}$. C'est la condition de la naissance de l'être humain profond et intérieur, immergé dans «l'opulence du silence $»^{54}$ et en même temps ouvert au Verbe. Malgré l'évidente tension entre l'extérieur et l'intérieur, le texte opte toujours pour la profondeur, pour le monastère métaphorique, pour cette clôture du titre, qui devient donc, paradoxalement, synonyme de liberté, mais aussi de beauté, de pureté de l'esprit, d'endroit propice à la création artistique.

Lorette Nobécourt, elle aussi, vit en quelque sorte comme une anachorète, s'imposant à elle-même les limites de sa clôture et vivant en accord avec son éthique personnelle exprimée dans les pages du roman analysé. Comme le remarque à juste titre Zoulim, « [s]on parcours est un exemple du mode d'être au monde d'un humain spirituel au XXI $\mathrm{XI}^{\mathrm{e}}$ siècle $»^{55}$.

\section{CONCLUSION}

\section{Heinz Nussbaumer constate que}

l'époque où nous vivons se caractérise par la coexistence de plusieurs contradictions, et cela aussi dans le domaine de la religion. D'une part, nous observons que les églises deviennent de plus en

49 Ibidem, p. 46.

50 Ibidem, pp. 46-47.

${ }^{51}$ Ibidem, p. 59.

${ }^{52}$ H. Nussbaumer, Odkryć w sobie mnicha. Pielgrzymując na górę Athos, trad. T. Sotowska, Wydawnictwo Pax, Warszawa 2008, p. 27.

53 Cela fait probablement écho aussi à la philosophie de Pascal qui indique les difficultés auxquelles l'homme est confronté au moment où il décide de séjourner quelque temps avec lui-même : « ...tout le malheur des hommes vient d'une seule chose, qui est de ne savoir pas demeurer en repos dans une chambre » (B. Pascal, Pensées, Le Livre de poche classique (Édition Sellier), Paris 1991, p. 121).

${ }^{54}$ L. Nobécourt, op. cit., p. 47.

55 C. Zoulim, op. cit., p. 140. L'hypothèse de l'identification de la narratrice à l'auteure demande une réflexion qui pourrait s'appuyer sur la théorie de l'expérience existentielle et mystique présentée, entre autres, par Jaspers. Vu que dans le présent article, une telle analyse ne serait que très restreinte et par conséquent superficielle, le problème, que je tiens pourtant à signaler, reste encore à examiner. 
plus vides, que le nombre de chrétiens engagés diminue, que, comme disent les sociologues, la religiosité disparaît. Mais de l'autre, les gens ressentent davantage le désir d'enracinement, d'appui, de profondeur, et aussi de mystique ${ }^{56}$.

Le roman de Nobécourt La Clôture des merveilles est un exemple d'une telle attitude. Il est l'expression de la nostalgie d'une vie intérieure profonde, mais en même temps, il se démarque d'une quelconque identification à la religion. La recherche spirituelle qui, pour Nobécourt et ses protagonistes, se réalise surtout dans l'activité de servir le Verbe, l'a menée à la rencontre avec la sainte médiévale, et par cela, lui a permis de découvrir le modèle de spiritualité bénédictine, qui a grandement influencé la chrétienté. Le choix d'Hildegarde a été certainement dicté par la communauté d'expérience des deux femmes, observée par Nobécourt, aussi bien au niveau spirituel et intellectuel qu'artistique. Le désir de plénitude a inspiré l'écrivaine à articuler son expérience à travers le personnage historique de la sainte. Il semble, pourtant, que le message du roman dépasse l'expérience individuelle (dans le cas contraire, il n'aurait été adressé qu'à un nombre limité de lecteurs), parce que la question de la plénitude, aussi bien dans le contexte religieux qu'en-dehors de celui-ci, se rapporte toujours à la question de l'humanité et de la grandeur de l'homme qui dépasse ses limites. L'essence de ce message est déjà signalée dans le titre du roman qui rappelle que les choses belles et pures, autrement dit les merveilles, se trouvent derrière les murs de la clôture qu'est l'être humain.

\section{HILDEGARD OF BINGEN - A POST-SECULAR SAINT? LA CLÔTURE DES MERVEILLES BY LORETTE NOBÉCOURT}

\section{Summary}

Lorette Nobécourt, belonging to the group of contemporary French writers who re-read biblical and hagiographical myths, may be regarded as one of the authors realizing to a greater or lesser extent the foundation of the post-secular thought. As Nobécourt's novel entitled La Cloture des merveilles. Une vie d'Hildegarde de Bingen (2013) can be seen as a representative example of a post-secular text, it has been analysed in the present paper. The aim of the article is to seek answers to the following questions: why has the contemporary French writer decided to present St Hildegard and how has the eponymous heroine been delineated in the novel? What is the relation between the Hildegard created by Nobécourt and the female narrator or, to take the argument one step further, what kind of spirituality do they represent? Finally, what does the word cloture used in the title mean to the contemporary laic reader? The present analysis of the novel, being an attempt to give answers to the above-mentioned questions, has been conducted on the basis of Charles Taylor's theory of "the place of fullness" and John A. McClure's concept of "new monasticism."

Key words: Lorette Nobécourt, St Hildegard of Bingen, post-secularism, new monasticism, the place of fullness, spirituality.

\footnotetext{
${ }^{56}$ H. Nussbaumer, op. cit., p. 16.
} 